ditions used to induce breakdown.

The LIDT for the diamond substrate was determined to be $1.20 \mathrm{~J} / \mathrm{cm}^{2}$ and 8.0 $\mathrm{J} / \mathrm{cm}^{2}$ for $200-\mathrm{ps}$ and $100-\mathrm{ns}$ pulse irradiation, respectively. The LIDT for diamond was found to be three times higher than for $\mathrm{ZnSe}$, and five to six times higher than for $\mathrm{Ge}$, depending on pulse length.

GREG KHITROV

\section{Ultraslow and Stored Light Observed in Crystalline Solid}

Ultraslow and stored light have been observed in a Pr-doped $\mathrm{Y}_{2} \mathrm{SiO}_{5}$ crystalline solid. A. Turukhin of JDS Uniphase and colleagues affiliated with the Massachusetts Institute of Technology, Texas A\&M University, the Electronics and Telecommunications Research Institute in South Korea, and the Air Force Research Laboratory at Hanscom Air Force Base demonstrated light speeds as slow as $45 \mathrm{~m} / \mathrm{s}$ and even light-pulse "stopping."

Slow-light experiments work by a dramatic reduction in group velocity. To significantly slow or "stop" light, it is desirable for a material to possess a sharp feature in its dispersion relation. Such featuresunusual in solid materials-can be achieved in certain insulators doped with rare-earth elements. The researchers in this study used Pr-doped $\mathrm{Y}_{2} \mathrm{SiO}_{5}$, which has previously been shown to exhibit electromagnetically induced transparency (EIT), and which has also been a crucial feature in atomic gases previously used for demonstrating ultraslow light.

As reported in the January 14 issue of Physical Review Letters, the crystal was optically pumped by coupling and probe laser fields and produced a "spectral hole," a region where the crystal is transparent to the laser field. Simultaneously, an auxiliary field was required to produce an absorbing "antihole." This auxiliary field not only controlled the probe field absorption, but also reduced the effective inhomogeneous linewidth the coupling field must overcome to achieve EIT. The group light velocity was measured by chopping the probe and determining the phase delay of the chopped signal.

Individual probe pulse slowing could also be measured. An advantage of this EIT-based technique was that the group delays depended mostly on the intensity of the coupling laser. This meant that the group light speed could be altered by changing the coupling laser intensity and that complex probe pulse shapes could be effectively slowed with bandwidth serving as the only real limitation. Finally, the researchers demonstrated "stopped light." This phenomenon occurs when the group velocity is decreased to zero while also reducing the coupling laser intensity to

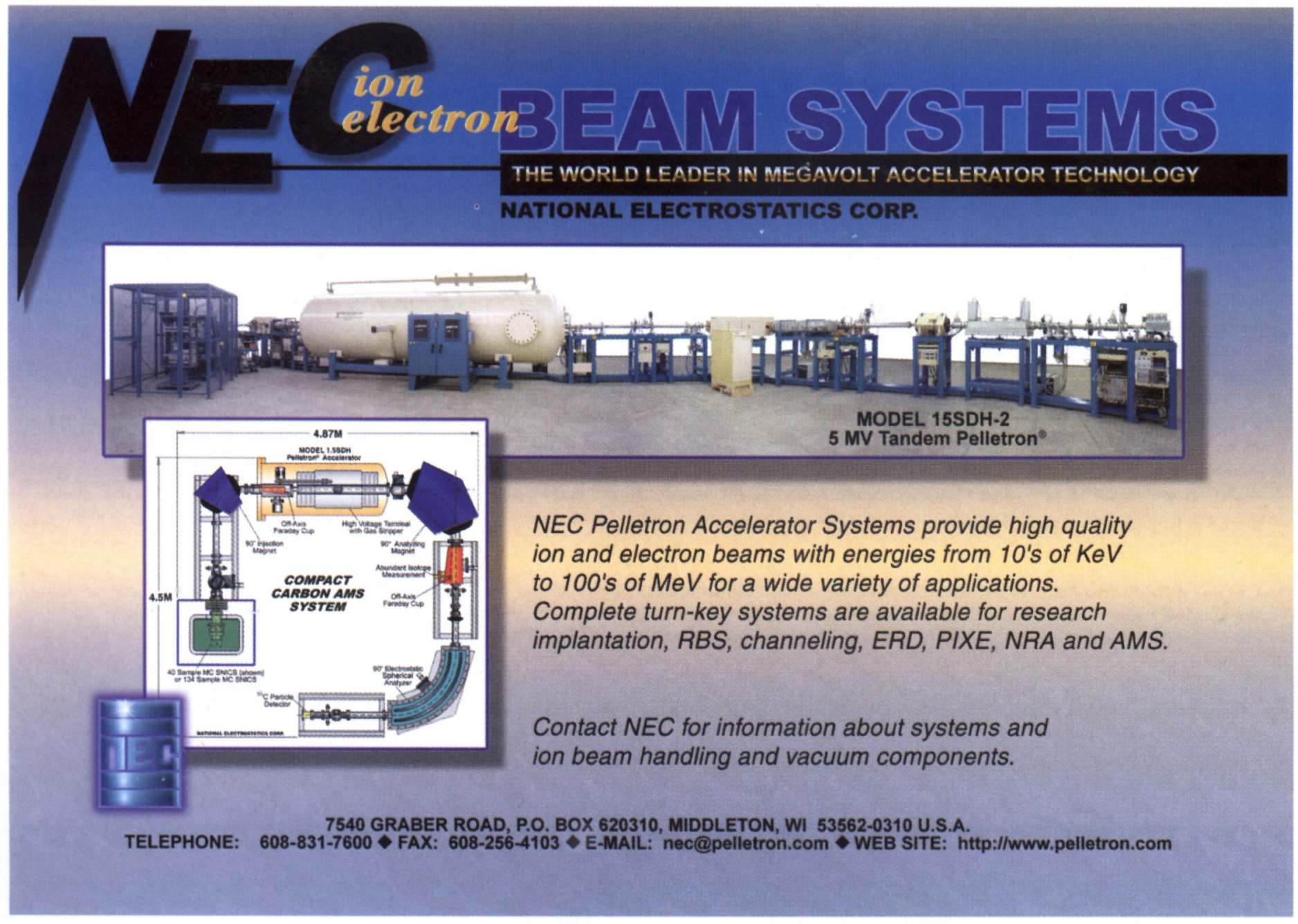


zero. In this way, the information in the probe pulse is "mapped" into the atomic coherence of the material, and can be read out subsequently by turning the coupling laser on again. The ability to store and recover the coherent light wave depends upon the spin coherence of the system as well as decoherence effects.

Since the initial "ultraslow" light observations in "cold" and "hot" atomic gases and subsequent extensions to "trapping" light, there has been considerable speculation regarding potential applications such as quantum computing, novel optical devices, and advanced measurement techniques. Nevertheless, the gaseous nature of the light-trapping medium has posed an obvious practical limitation for a variety of these uses. The researchers believe that their use of a solid-state medium opens the door to potential practical applications that had previously not been feasible from a materials perspective. However, all of the work reported here has been performed on samples held at a temperature of $5 \mathrm{~K}$. Practical applications may require operation at much higher temperatures.

EMILY JARVIS

\section{Electromagnetic Field Guides Flow through Microfluidic Networks}

Nonspecific binding to channel walls and particle clogging of microchannels are key obstacles that limit the application of microfluidic lab-on-a-chip technologies. In the February 25 issue of Applied Physics Letter, researchers G. Zabow and colleagues at Harvard University described a technique that allowed guided flow of particles through microchannels, even through those with arbitrary geometries, without particle adhesion to channel walls.

The technique was an electromagnetic guided approach to attract transported particles toward the channel centers and eliminate particle adhesion to channel walls, control particle spreading due to Poiseuille flows, prevent gravitational settling, and provide a means for rapid particle separation. A net force was applied on the particles and was defined by the applied electromagnetic force in conjunction with surface-tension constraints inherent to the microfluidic capillary network. The researchers said that for interfaces (liquid-liquid or liquid-gas) with a nonzero curvature, or a field with a nonzero curvature over the surface defined by the liquid interface, net forces parallel to the surface resulted that could be exploited to direct particle flow. Such curved fluid interfaces were achieved by forming fluid channels from complementary molds with the bottom and top half hydrophilic and hydrophobic, respectively. The pressure difference across the fluid surface set the liquid-wall contact angle, $\theta$, and surface curvature and was readily controlled through fluid flow velocity, gas pressure, or channel width. For fluid contact angles $\theta>90^{\circ}$, the interface bulged upward in the center, creating a local electromagnetic potential minimum where the particles accumulated. Conversely, for $\theta<90^{\circ}$, the interface lowered in the center, and particles moved toward the channel walls. Rapid particle separation of the ongoing stream was possible by switching from $\theta>90^{\circ}$ to $\theta<90^{\circ}$.

The researchers considered an annular ring with a large radius to prove their claims both numerically and experimentally. They modeled the fluid surface profile by solving a differential equation that included the effects of the difference in density of the fluids, the fluid surface tension, and gravitational effects. They then overlaid the profile with a density plot of applied magnetic field. To test the theory, experiments were conducted with a transparent poly(dimethylsiloxane) ring channel (4-mm width) and 4.5- $\mu \mathrm{m}$-diameter superparamagnetic beads. The results showed excellent agreement with theoretical predictions, with the same beads flowing at the channel center and around the

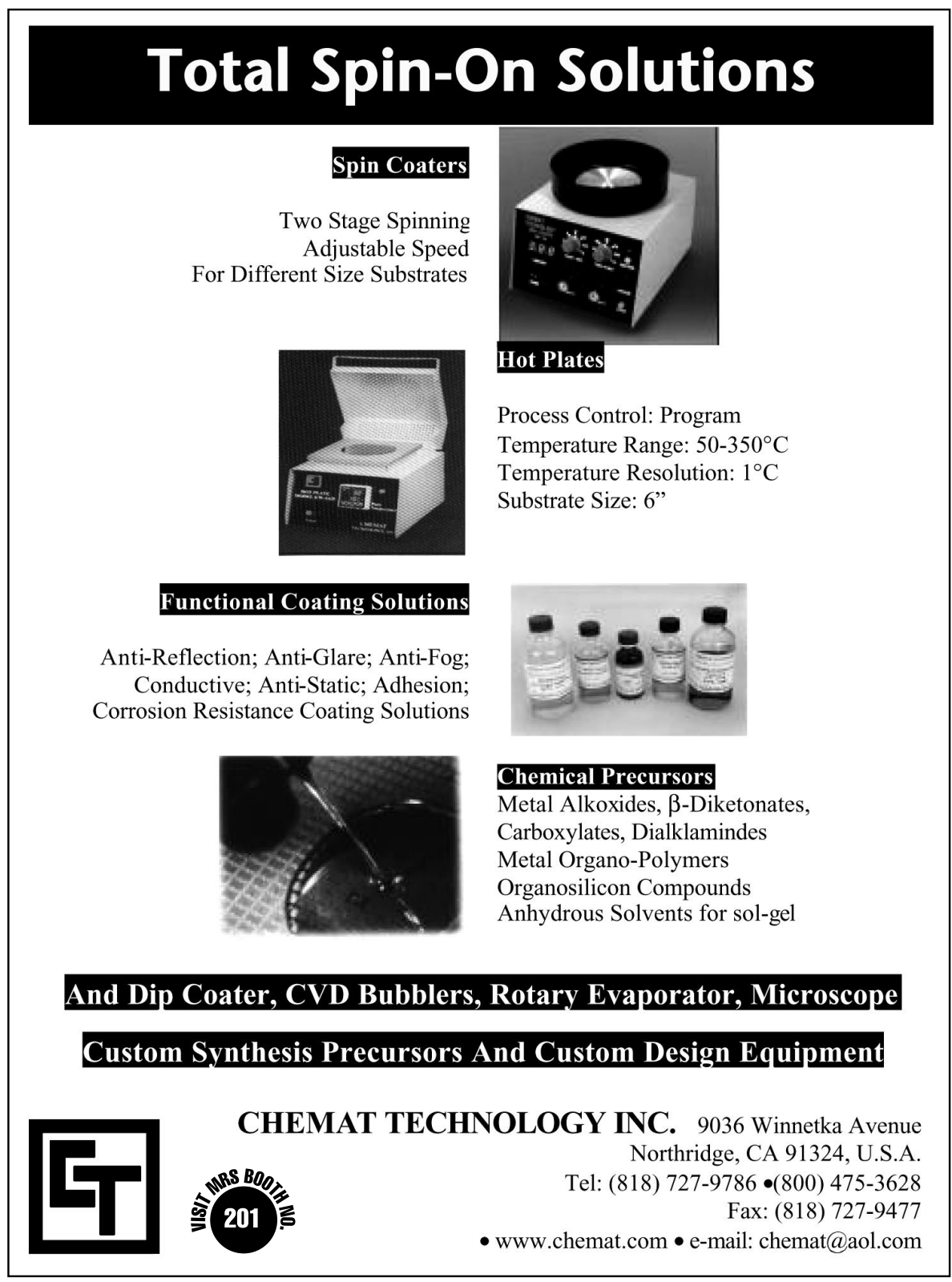

Circle No. 4 on Inside Back Cover 